\title{
Creatine kinase-(MB) and hepcidin as candidate biomarkers for early diagnosis of pulmonary tuberculosis: a proof-of-concept study in Lambaréné, Gabon
}

\author{
Paulin N. Essone ${ }^{1,2,3}\left(\mathbb{D} \cdot\right.$ Bayode R. Adegbite ${ }^{1,2,4} \cdot$ Marien J. M. Mbadinga $^{1} \cdot$ Armel V. Mbouna $^{1} \cdot$ \\ Fabrice Lotola-Mougeni ${ }^{1}$. Ayodele Alabi ${ }^{1,5}$. Jean R. Edoa ${ }^{1,4}$ - Bertrand Lell ${ }^{1,6}$. Abraham S. Alabi ${ }^{1,2}$. \\ Ayola A. Adegnika ${ }^{1,2,5}$. Michael Ramharter ${ }^{1,7}$. Joel F. D. Siawaya ${ }^{3} \cdot$ Martin P. Grobusch ${ }^{1,2,4} \cdot$ Peter G. Kremsner $^{1,2}$. \\ Selidji T. Agnandji ${ }^{1,2,8}$
}

Received: 8 November 2021 / Accepted: 15 January 2022 / Published online: 8 February 2022

(C) The Author(s) 2022

\begin{abstract}
Background The present study aimed to evaluate the diagnostic utility of creatine kinase-MB (CK-MB), hepcidin (HEPC), phospholipase A2 group IIA (PLa2G2A), and myosin-binding protein C (MYBPC1) for tuberculosis (TB). These four biomarkers are differentially regulated between quiescent Mycobacterium tuberculosis (Mtb) infected individuals (nonprogressors to TB disease) and Mtb-infected TB disease progressors 6 months before the onset of symptoms.

Methods We enrolled samples from patients experiencing moderate-to-severe pulmonary infections diseases including $23 \mathrm{~TB}$ cases confirmed by smear microscopy and culture, and 34 TB-negative cases. For each participant, the serum levels of the four biomarkers were measured using ELISA.

Results The levels of CK-MB and HEPC were significantly reduced in patients with active TB disease. CK-MB median level was $2045 \mathrm{pg} / \mathrm{ml}(1455-4000 \mathrm{pg} / \mathrm{ml})$ in active TB cases and $3245 \mathrm{pg} / \mathrm{ml}(1645-4000 \mathrm{pg} / \mathrm{ml})$ in non-TB pulmonary diseases. Using the receiver operating characteristic curve (ROC) analysis, HEPC and CK-MB had the Area Under the Curve (AUC) of $79 \%$ (95\% CI 67-91\%) and 81\% (95\% CI 69-93\%), respectively. Both markers correlated with TB diagnosis as a single marker. PLa2G2A and MYBPC1 with AUCs of 48\% (95\% CI 36-65\%) and 62\% (95\% CI 48-76\%) did not performed well as single biomarkers. The three markers' model (CK-MB-HEPC-PLa2G2A) had the highest diagnostic accuracy at $82 \%(95 \%$ CI 56-82\%) after cross-validation.

Conclusion CK-MB and HEPC levels were statistically different between confirmed TB cases and non-TB cases. This study yields promising results for the rapid diagnosis of TB disease using a single marker or three biomarkers model.
\end{abstract}

Keywords Biomarkers $\cdot$ Tuberculosis $\cdot$ Diagnosis $\cdot$ Creatine kinase-MB $\cdot$ Hepcidin

\section{Introduction}

About 10 million individuals develop tuberculosis (TB) every year, causing 1.3 million deaths per year [1]. The limited performance and reduced accessibility of diagnostic methods contribute to the high morbidity and mortality of

Paulin N. Essone, Bayode R. Adegbite co-first authors.

Paulin N. Essone

paulin.essone@cermel.org

Selidji T. Agnandji agnandjis@cermel.org

Extended author information available on the last page of the article
TB [2]. Immunodiagnostic techniques such as point-of-care tests could substantially improve TB control. The tuberculin skin test (TST) and interferon (IFN)-g release assays (IGRA) cannot distinguish between TB disease and other respiratory infections [3]. Cytokine secretion profiles derived from antigen stimulation have been evaluated as immune diagnostic tests for TB. Various Mycobacterium tuberculosis (Mtb) antigens [4] and cytokine/chemokines other than IFN-g have been studied [5, 6]. Overnight incubation and other technical hurdles requested by the Mtb antigen-stimulated cytokine biosignatures test may be challenging in resource-limited settings. The identification of host markers from serum samples that have a more specific action in the host responses for 
the development of a rapid point-of-care test for TB disease remains, as of today, a by-and-large unmet medical need.

In a recent prospective cohort study of about 6000 quiescent Mtb-infected individuals, the prognostic utility of more than 3000 plasma proteins was evaluated [7]. A total of 42 individuals developed TB disease within 2 years (TB progressors) in this study. Proteins were quantified in plasma with a highly multiplexed proteomic assay (SOMAscan); 361 proteins including creatine kinase myocardial band (CK-MB), hepcidin (HEPC), phospholipase A2 group IIA (PLa2G2A) and myosin-binding protein C (MYBPC1) were differentially expressed between TB progressors and nonprogressors before the onset of symptoms.

Hepcidin is a regulator of iron metabolism, which controls the expression of the transmembrane protein ferroportin. This transmembrane protein reduces the plasma level of iron by increasing iron intake by macrophages and limiting iron absorption during food intake [8]. A high plasma level of hepcidin will increase ferroportin expression and induces high concentration of iron within macrophages. A reduced hepcidin level will lead to low iron quantity inside macrophages and prevents Mtb growth [9-11]. An elevated hepcidin level is associated with disseminated TB disease, anaemia, and poor prognosis in patients with HIVassociated tuberculosis [12]. CK-MB is routinely used as a biomarker of myocardial infarction. An elevated level of this biomarker is an indicator of cardiac muscle damage [13]. The level of cardiac muscle damage during TB disease is unknown, although myopericarditis is common in TB patients [14]. PLa2G2A is an extracellular enzyme catalyzing the hydrolysis of the sn-2 fatty acid acyl ester bond of phosphoglycerides[15]. It is thought to participate in the regulation of phospholipid metabolism in biomembranes. A high concentration of this protein has been associated with coronary heart disease, rheumatoid arthritis and asthma. [16, 17]. MYBPC1 is a smooth skeletal muscle-specific enzyme. It plays an essential role in muscle contraction by recruiting muscle-type creatine kinase to myosin filaments[18]. This protein has been associated with breast cancer prognosis[19]. The functions of PLa2G2A and MYBPC1 during TB have not been studied. The present study aims to evaluate the secretion levels of CK-MB, HEPC, PLa2G2A and MYBPC1 in active TB disease and other pulmonary disease patients to determine their diagnostic utility.

\section{Materials and methods}

\section{Study design}

We conducted a cross-sectional study, enrolling samples received at the Centre de Recherches Médicales de Lambaréné from January 2019 to December 2020 for TB testing.
Samples from participants experiencing TB-suggestive symptoms and providing written informed consent were enrolled to evaluate the diagnostic utility of selected biomarkers in resource-constrained settings using the method developed in previous studies [20-22].

\section{Study participants and procedure}

Adults ( $\geq 18$ years) with TB-suggestive symptoms who attended the TB laboratory of CERMEL or were hospitalised in one of the Lambaréné hospitals were consecutively screened. Patients who were unable to provide informed consent were excluded. For volunteers, written informed consent was obtained, and a structured questionnaire addressing sociodemographic and clinical information was administered. All patients provided two sputum samples (one during the outpatient/inpatient attendance and one in the early morning the day after) as suggested by the national tuberculosis control programme and described elsewhere [23]. Participants with positive smear microscopy and positive Mtb culture results were defined as TB cases, while non-TB patients were defined as smear- and culture-negative. Whole blood collected in a dried tube for each participant was centrifuged, and serum was collected into several aliquots and stored at $-80^{\circ} \mathrm{C}$. The individual biomarker secretion levels were compared between confirmed TB cases and non-TB patients.

\section{Biomarkers selection process}

The four biomarkers of interest in our study were selected from a list of proteins reported by Penn-Nicholson et al. [7] as potential predictors of TB disease in participants with quiescent Mtb. More than 6000 Mtb infected HIV-negative participants were followed for 2 years and samples actively and passively collected from onset of TB disease. Participants were categorized as TB progressors if they developed TB during the follow-up period, and as TBnon-progressors if they did not develop TB disease. The expression profiles of more than 3000 proteins were compared between TB disease progressors and non-progressors using SomaLogic technology [7]. This platform is based on modified-aptamer binding technology (SOMAmers) that bind to their respective target proteins to determine their presence and quantity in a sample. The performances of each protein, including $\mathrm{p}$ value, fold change, and False Discovery Rate (FDR), are provided in Supplementary Table 1. Applying strict selection criteria, we selected proteins with a significant $p$ value, a fold change difference of a least 1.4 and a False Discovery Rate (FDR) of less than 0.05 as potential TB diagnostic candidates. A reduced list of 31 proteins differentially expressed between TB progressors and non-progressors was obtained (Supplementary Table 1). We selected four biomarkers (CK-MB, 
HEPC, PLa2G2A and MYBPC1) for evaluation in this pilot study due to their potential role in expressing anti-TB properties of macrophages (the most abundant cell population in tuberculous granuloma) [24].

\section{ELISA}

The serum levels of CK-MB, HEPC, PLa2G2A and MYBPC1 were measured using ELISA kits (MyBioSource, San Diego, USA). ELISAs were performed according to the manufacturer's instructions. Diluted standards, blanks and $5 \mathrm{X}$ diluted samples were added into the appropriate pre-coated plates. The plates were incubated for $90 \mathrm{~min}$ at $37{ }^{\circ} \mathrm{C}$. A hundred (100) $\mu \mathrm{l}$ of detection solution A was added to each well plate and set for $45 \mathrm{~min}$. The plates were washed three times before adding $100 \mu \mathrm{l}$ of detection solution B and incubation for an additional $45 \mathrm{~min}$. The plates were then washed five times, and $90 \mu \mathrm{l}$ of substrate solution was added and incubated in the dark for $15 \mathrm{~min}$. $50 \mu \mathrm{l}$ of stop solution was added to each well and read at $45 \mathrm{~nm}$ using the ELISA reader.

\section{Statistical analysis}

Comparison of the two study groups (TB vs no TB) was performed using the Chi-square (Chi2) for women and men proportions, and Kruskall-Wallis test for the distribution of age and BMI. The accuracy of all biomarkers for the diagnosis of TB disease was estimated by performing receiver operator characteristics (ROC) curve analysis. The utility of combinations of biomarkers for TB diagnostic was investigated by performing best subsets general discriminant analysis (GDA), with leave-one-out crossvalidation (where all data were individually validated). Data were analysed using the $\mathrm{R}$ software, version 4.0.3 and GraphPad prism, version 5.0 (GraphPad Software, San Diego, CA, USA).

\section{Results}

\section{Study participants}

Fifty-seven (57) participants were enrolled in this study, including 23 confirmed TB cases and 34 non-TB cases (Table 1). The median age of included participants was 44 [IQR 22-59]. Weight, sex and height were evenly distributed between the two study groups.

\section{The utility of single biomarkers in discriminating TB from alternative pulmonary diseases}

The serum levels of MYBPC1, PLa2G2A, CK-MB and HEPC were compared between confirmed TB cases and pulmonary infections other than TB. All four biomarker candidates were detected in both study groups (Fig. 1). Serum levels of CK-MB and HEPC were significantly reduced in TB cases $(P<0.001)$ compared to non-TB. The diagnostic accuracy of the four biomarkers was evaluated by ROC curve analysis. The area under the ROC curve (AUC) of MYBPC1 and PLa2G2A were 0.48 and 0.62, respectively, indicating a low diagnostic accuracy. PLa2G2A has the poorest diagnostic utility with sensitivity and specificity around $50 \%$ (cut off $=1.19 \mathrm{ng} / \mathrm{ml}$ ). With an AUC of 0.62 (62\%), MYBPC1 has a sensitivity of $70 \%$ but a reduced specificity of $61 \%$ for a cut-off at $26.98 \mathrm{ng} / \mathrm{ml}$. CK-MB and HEPC yielded AUCs of $81 \%$ and $79 \%$, respectively (Fig. 1, Table 2). For a selected cut-off at $515.9 \mathrm{ng} / \mathrm{ml}$ (optimal sensitivity and specificity), CK-MB had a sensitivity of $78.3 \%$ and specificity of $73.5 \%$, respectively. The sensitivity and specificity of HEPC were $87 \%$ and $64 \%$, respectively, for a cut-off at $130.6 \mathrm{ng} / \mathrm{ml}$.

\section{The utility of 2- to 3-biomarker models in the rapid diagnosis of TB disease}

The diagnostic accuracy of TB disease was evaluated using several models. CK-MB and HEPC had respective AUCs of
Table 1 Demographic characteristics of study participants

\begin{tabular}{lllll}
\hline & All & No TB & TB \\
$N=62$ & $N=36$ & $N=26$ & $P$ value \\
\hline Gender & & & & 0.900 \\
Women $(N, \%)$ & $28(45.2)$ & $17(47.2)$ & $11(42.3)$ & \\
Men $(N, \%)$ & $34(54.8)$ & $19(52.8)$ & $15(57.7)$ & \\
Age median [IQR] & $43.9[22.2-59.2]$ & $49.2[23.2-62.6]$ & $34.1[22.3-49.6]$ & 0.077 \\
BMI, median $[\mathrm{IQR}]$ & $20.1[16.7-22.1]$ & $20.9[17.2-24.3]$ & $19.7[16.5-20.9]$ & 0.180 \\
\hline
\end{tabular}

$\mathrm{Chi}^{2}$ test was used to compare women and men proportion, and Kruskall-Wallis test was used to compare the distribution of the Age and the BMI acconding to TB status

$B M I$ body mass index, $I Q R$ interquartile range 


\section{CKMB}
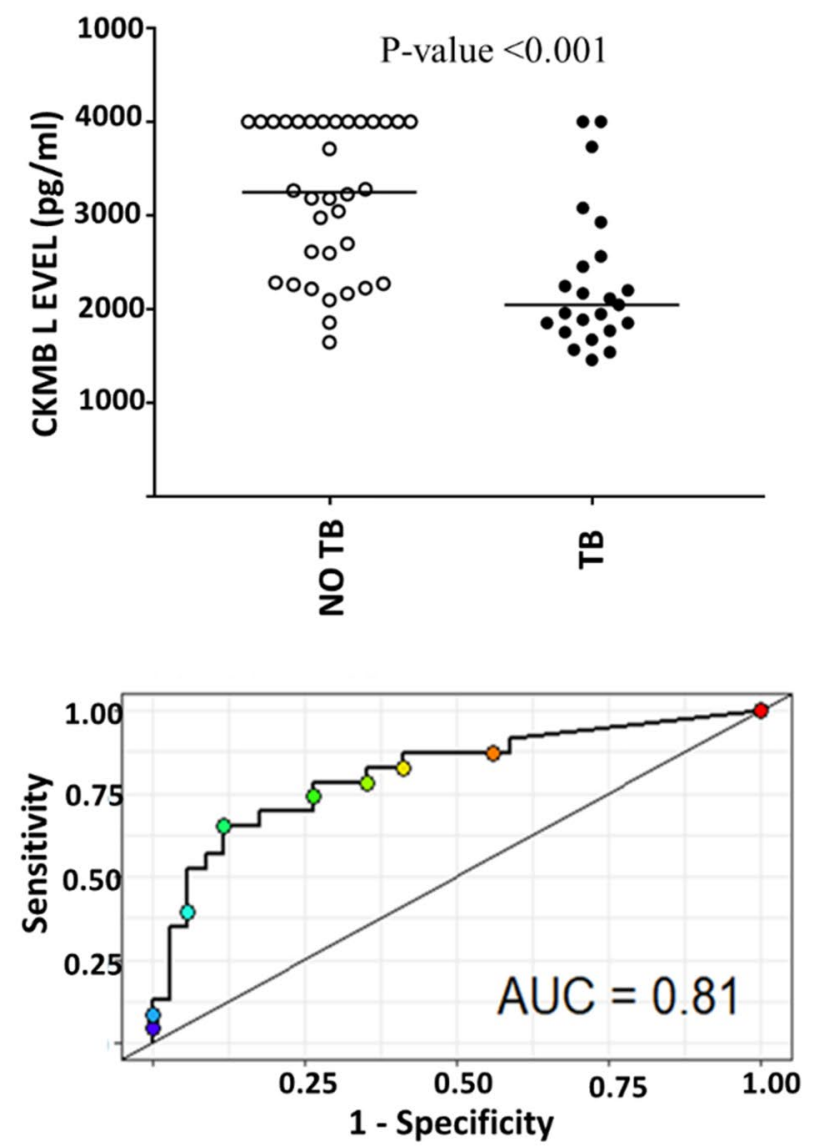

Fig. 1 Scatter-dot plots and ROC curves of analytes discriminating TB disease to alternative pulmonary diseases. Representative dot plots showing the levels of analytes in serum and ROC curves for
HEPC
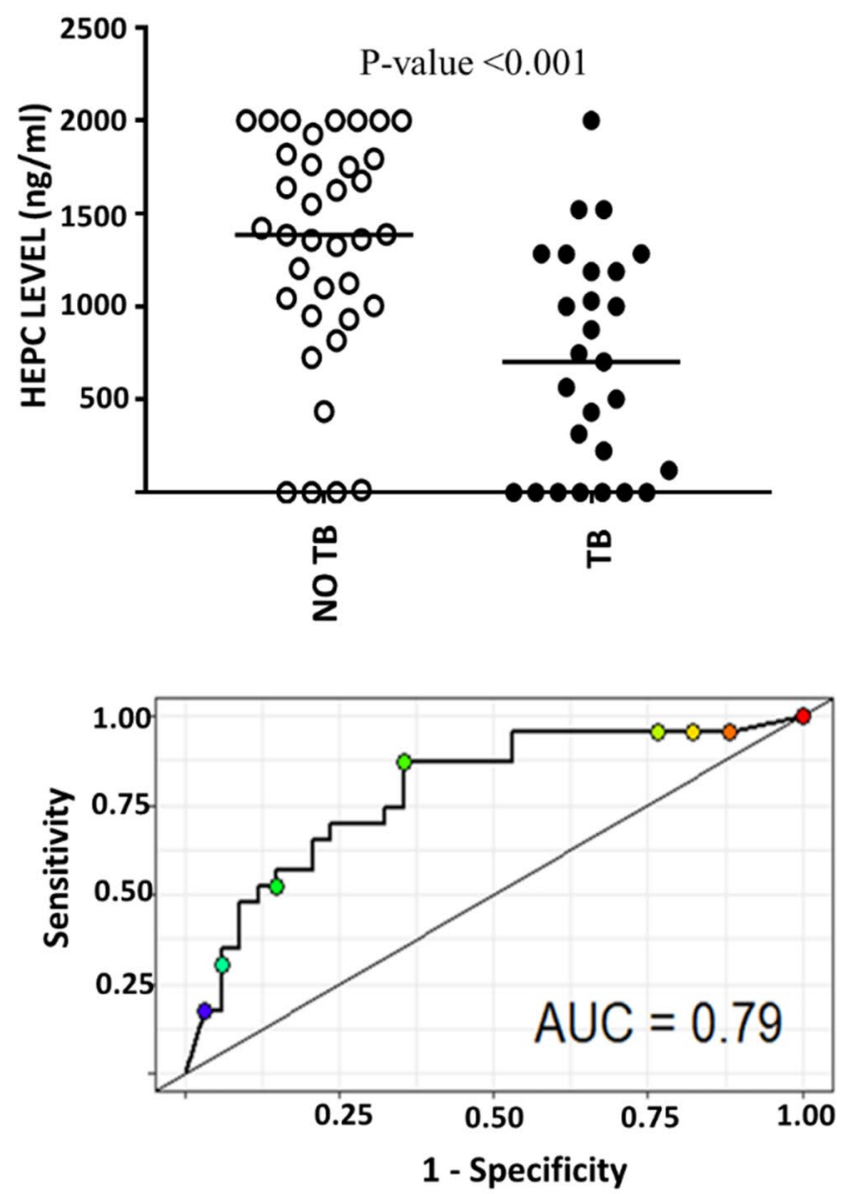

accuracy in the diagnosis of TB disease. Error bars in the dot plots represent the median analyte levels

Table 2 Diagnostic potential of single host markers

\begin{tabular}{llllllcr}
\hline Host markers & TB (range) & No TB (range) & $P$-value & AUC \% (95\% CI) & $\begin{array}{l}\text { Sensitivity (95\% } \\
\text { CI) }\end{array}$ & $\begin{array}{l}\text { Specificity (95\% } \\
\text { CI) }\end{array}$ & Cut off value \\
\hline MYBPC1 (ng/ml) & $170(70-500)$ & $125(70-500)$ & 0.3 & $62(48-76)$ & $70(50-86)$ & $61(43-77)$ & $>26.98$ \\
PLa2G2A (pg/ml) & $0(0-2000)$ & $0(0-2000)$ & 0.8 & $48(36-65)$ & $48(29-68)$ & $56(38-72)$ & $>1.19$ \\
CK-MB (pg/ml) & $2045(1455-4000)$ & $3245(1645-4000)$ & $<\mathbf{0 . 0 0 1}$ & $\mathbf{8 1}(69-93)$ & $78.3(56-93)$ & $73.5(56-87)$ & $<515.9$ \\
HEPC $(\mathrm{ng} / \mathrm{ml})$ & $375(0-1000)$ & $700(0-1000)$ & $<\mathbf{0 . 0 0 1}$ & $\mathbf{7 9}(67-91)$ & $87(66-97)$ & $64(46-80)$ & $<130.6$ \\
\hline
\end{tabular}

Median levels (and ranges in parenthesis) of serum analytes and their accuracy in the diagnosis of TB disease as a single host marker. $P$ values were calculated using the Mann Whitney $U$ test

$A U C$ area under the receiver operator characteristics curve, $95 \%$ CI 95\% confidence interval

$81 \%$ and $79 \%$ as single markers. When the two most accurate single biomarkers CK-MB and HEPC were tested as a model, their diagnostic accuracy increased to $82 \%$. After leave-one-out cross-validation analysis, the accuracy of this model was found to be $75.4 \%$. The CK-MB-HEPC model successfully classified (after leave-one-out cross-validation)
76.5\% non-TB and $73.9 \%$ TB cases, respectively (Fig. 2 and Table 3). The diagnostic accuracy of MYBPC1 was low as a single biomarker. This biomarker was associated with CK-MB in a 2-biomarkers model, and it had the highest diagnostic accuracy (AUC of 85\%) of all 2-biomarkers models. However, the diagnostic accuracy of this model was 

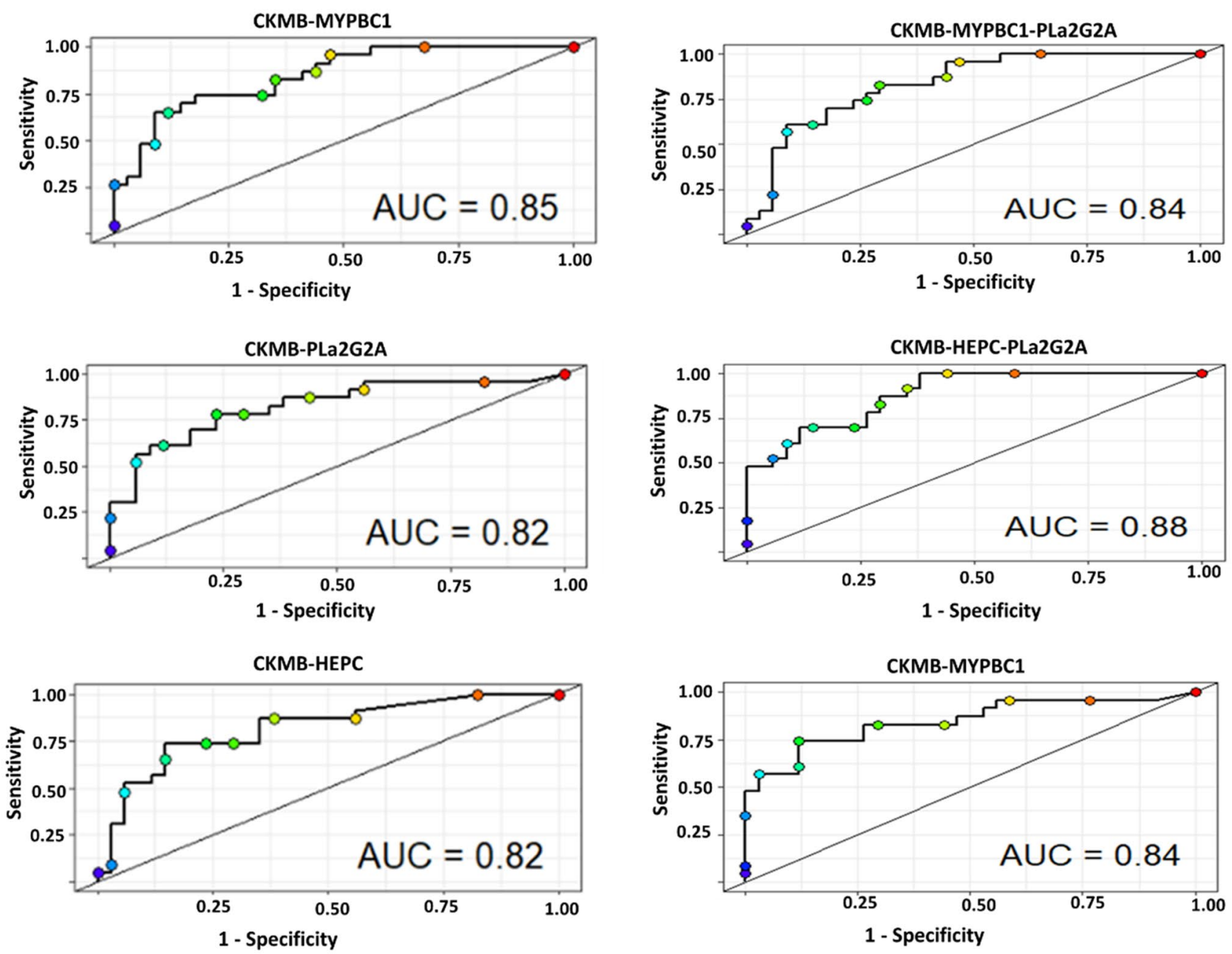

Fig. 2 ROC curves analysis of the most discriminative two and tree markers models

Table 3 Utility of combination of analytes in the diagnosis of TB disease

\begin{tabular}{|c|c|c|c|c|c|c|c|}
\hline \multirow[t]{2}{*}{ Combinations } & \multicolumn{3}{|c|}{ Leave-one-out cross validation } & \multirow[t]{2}{*}{ AUC } & \multirow[t]{2}{*}{ Wilks } & \multirow[t]{2}{*}{$f$} & \multirow[t]{2}{*}{$P$-value } \\
\hline & TB cases $(\%)$ & No TB $(\%)$ & Accuracy $(\%)$ & & & & \\
\hline CKMB, HEPC & 73.9 & 76.5 & $75.4[62.2-85.9]$ & 82 & 0.29 & 65.1 & $<0.001$ \\
\hline MYBPC1, HEPC & 47.8 & 82.4 & $68.4[54.8-80.1]$ & 79 & 0.47 & 30.4 & $<0.001$ \\
\hline MYBPC1, CKMB & 73.9 & 67.6 & $70.2[56.6-81.6]$ & 85 & 0.28 & 70.9 & $<0.001$ \\
\hline PLa2G2A, CKMB, HEPC & 73.9 & 88.2 & $82.5[70.1-91.3]$ & 84 & 0.34 & 33.8 & $<0.001$ \\
\hline MYBPC1, CKMB, HEPC & 69.6 & 79.4 & $75.4[62.2-85.9]$ & 88 & 0.33 & 35.6 & $<0.001$ \\
\hline MYBPC1, PLa2G2A, HEPC & 47.8 & 85.3 & $70.2[56.6-81.6]$ & 78 & 0.48 & 19.3 & $<0.001$ \\
\hline MYBPC1, PLa2G2A, CKMB & 73.9 & 76.5 & $75.4[62.2-85.9]$ & 84 & 0.29 & 42.7 & $<0.001$ \\
\hline
\end{tabular}

significantly reduced to $70.2 \%$ after leave-one-out crossvalidation analysis.

The AUC of 3-biomarker models varied between 78 and $88 \%$. The CK-MB-HEPC-MYBPC1 model was the most accurate with an AUC of $88 \%$ but dropped to an AUC of $75.4 \%$ after leave-one-out cross-validation analysis.

The CK-MB-HEPC-PLa2G2A model had an AUC above $82 \%$ after leave-one-out cross-validation analysis. This 3-biomarker model successfully classified $88.2 \%$ of non-TB cases and $73.9 \%$ of active TB diseases. 


\section{Discussion}

Rapid diagnosis of active TB remains an unmet medical need due to the lack of good biomarkers. The current study shows that CK-MB and HEPC are differentially expressed in active TB cases compared to patients with pulmonary infections other than TB.

Previous studies have shown promising results with novel Mtb antigen-stimulated growth factors and cytokines, including epidermal growth factor (EGF), tumour necrosis factor-alpha (TNF- $\alpha$ ), vascular endothelial growth factor (VEGF), interferon-gamma (IFN- $\gamma$, IL-12 and IL10 [5, 25]. These biomarkers were differentially expressed when comparing TB cases to healthy household contacts in a 7-day assay. In an overnight assay with alternative pulmonary diseases as controls (instead of healthy controls), none of these markers could differentiate TB from other pulmonary diseases $[20,26]$. These studies have shown the limitation of cytokines/chemokines as biomarkers for the rapid diagnosis of TB disease.

The current study illustrates the utility of circulating CK-MB and HEPC levels for TB diagnosis. CK-MB is present in myocardial cells and is the most specific enzyme for clinical diagnosis of myocardial injury [27]. This enzyme has also been identified in patients presenting with a wide range of noncardiac diseases [28-30]. Although the link between CK-MB and infectious diseases is not fully understood, it has been detected in patients with infectious diseases, including dengue infection, in the absence of heart disease or myositis [31, 32]. The secretion level of CK-MB in pulmonary diseases has been investigated. A study performed in Japan investigating the level of CK-MB in different lung carcinomas has shown a low secretion level of this marker [33]. Several studies showed increased secretion of CK-MB in patients with Severe Acute Respiratory Syndrome Coronavirus-2 (SARS-CoV-2) [34]. The secretion level of this enzyme was associated with the severity and case fatality rate during SARS-CoV-2 infection $[35,36]$. Increased levels of CK-MB in SARS-CoV-2 patients with mild infection has also been reported [37]. CK-MB is not specifically secreted during TB disease, but its expression profile could help the rapid diagnosis of active TB compared to alternative pulmonary infections.

Hepcidin (HEPC) is an antimicrobial-like peptide hormone; defined as the master regulator of iron metabolism. We found a reduced level of this hormone in TB patients compared to pulmonary infections other than TB patients in the current study. Mtb infection of mice resulted in decreased levels of hepcidin [38]. Hepcidin regulates iron through modulation of the iron transporter ferroportin highly expressed in macrophages. Hepcidin reduces the circulating level of iron by increasing iron intake into macrophages and limited dietary iron absorption $[8,39]$. Iron is essential for bacteria growth. A high concentration of hepcidin during Mtb infection may lead to a high concentration of iron in infected macrophages and favour intracellular growth of the bacteria, suggesting a potential role of HEPC in regulating the anti-TB function of lung-resident macrophages. Hepcidin is stable in healthy individuals, and it is not influenced by age. An elevated level of hepcidin in HIV-infected individuals is a prognostic biomarker of TB in this population [12]. A high serum concentration of this biomarker has been positively correlated to a late time to Mtb culture conversion [9]. The interpretation of hepcidin levels during TB disease may depend on malaria infection in malaria-endemic areas. Data have shown that febrile malaria increases the serum level of hepcidin while severe and complicated malaria is associated with reduced plasma levels in African children [9]. Particular attention should be paid to the co-morbidity of TB-malaria when interpreting hepcidin results. The present study shows the potential utility of hepcidin and CK-MB as biomarkers for diagnosing TB disease. Although these biomarkers are secreted in most pulmonary conditions, they may have a particular secretion profile during TB. They could help in the development of TB rapid point-of-care tests.

Healthy participants and quiescent Mtb infected individuals were not enrolled in our study. Their presence may have added information rendering interpretation of our results possibly less straightforward. However, quiescent Mtb infection is not routinely tested in high-endemic countries like Gabon and has no impact on public health strategy. Despite these potential limitations, we think that the study design responded to the study's main objective to assess the diagnostic utility of the selected biomarker.

The selection of four biomarkers out of 31 identified in the predictive utility study may appear arbitrary, but was based on their high value as disease progression markers and on our assumption that they play roles in the anti-TB functions of the lung-resident macrophages. Further steps towards a large and multicenter validation trial require (1) a mechanistic confirmatory study of the roles of CK-MB and hepcidin in the regulation of anti TB functions of lung-resident macrophages. (2) a study that assesses the diagnostic utility, mode of action and standard ranges of an additional list of biomarkers.

\section{Conclusion}

The present study demonstrates potential, warranting further exploration of CK-MB and hepcidin in the rapid diagnosis of TB disease. CK-MB and hepcidin were differentially expressed in active TB diseases when compared to 
pulmonary diseases other than TB, presenting a good AUC as a single marker. The current study is a proof-of-concept in which the results require validation in a more extensive and multicenter study population. The diagnostic performance of of these biomarkers in specific population like smearnegative TB and HIV-TB patients need to be investigated.

Supplementary Information The online version contains supplementary material available at https://doi.org/10.1007/s15010-022-01760-8.

Author contributions PNE, BRA and STA designed the study. BRA, JRE, AA, AVM and ASA managed participants and data. PNE, MJMM performed the experiments. FLM, PNE, BL, STA and JLDS analysed the data. PNE, MJMM, FLM wrote the first draft. STA, MPG, AAA, MR, PGK and JLDS substantially reviewed an improve this manuscript.

Funding Open Access funding enabled and organized by Projekt DEAL. This work was supported by European and Developing Countries Clinical Trials Partnership (EDCTP-TMA-SF-1946-VARSAF).

Availability of data and material All original data will be made available on request by the correcponding authors.

\section{Declarations}

Conflict of interest The authors declare no conflict of interest.

Ethics statement Following scrutiny and endorsement by the CERMEL Scientific Review Committee, the Institutional ethic committee of CERMEL approved this study. All study participants gave written informed consent for participation in the study.

Open Access This article is licensed under a Creative Commons Attribution 4.0 International License, which permits use, sharing, adaptation, distribution and reproduction in any medium or format, as long as you give appropriate credit to the original author(s) and the source, provide a link to the Creative Commons licence, and indicate if changes were made. The images or other third party material in this article are included in the article's Creative Commons licence, unless indicated otherwise in a credit line to the material. If material is not included in the article's Creative Commons licence and your intended use is not permitted by statutory regulation or exceeds the permitted use, you will need to obtain permission directly from the copyright holder. To view a copy of this licence, visit http://creativecommons.org/licenses/by/4.0/.

\section{References}

1. WHO I Global tuberculosis report 2018 [Internet]. WHO. Available 2019 Aug 29, from http://www.who.int/tb/publications/ global_report/en/. Accessed 29 Aug 2019

2. Afsar I, Gunes M, Er H, Sener AG. Comparison of culture, microscopic smear and molecular methods in diagnosis of tuberculosis. Rev Esp Quimioter. 2018;31:435-8.

3. Perez-Velez CM, Marais BJ. Disease burden and recent epidemiologic shifts. Tuberculosis. 2012. https://doi.org/10.1056/NEJMr a1008049.

4. Ngabonziza JCS, Ssengooba W, Mutua F, Torrea G, Dushime A, Gasana M, Andre E, Uwamungu S, Nyaruhirira AU, Mwaengo D, Muvunyi CM. Diagnostic performance of smear microscopy and incremental yield of Xpert in detection of pulmonary tuberculosis in Rwanda. BMC Infect Dis. 2016. https://doi.org/10.1186/ s12879-016-2009-x.

5. Chegou NN, Essone PN, Loxton AG, Stanley K, Black GF, van der Spuy GD, van Helden PD, Franken KL, Parida SK, Klein MR, Kaufmann SHE, Ottenhoff THM, Walzl G. Potential of host markers produced by infection phase-dependent antigen-stimulated cells for the diagnosis of tuberculosis in a highly endemic area. PLoS ONE. 2012. https://doi.org/10.1371/journal.pone.0038501.

6. Essone PN, Kalsdorf B, Chegou NN, Loxton AG, Kriel M, Preyer R, Ernst M, Walzl G, Lange C. Bifunctional T-cell-derived cytokines for the diagnosis of tuberculosis and treatment monitoring. Respiration. 2014;88:251-61. https://doi.org/10.1159/00036 5816.

7. Penn-Nicholson A, Hraha T, Thompson EG, Sterling D, Mbandi SK, Wall KM, Fisher M, Suliman S, Shankar S, Hanekom WA, Janjic N, Hatherill M, Kaufmann SHE, et al. Discovery and validation of a prognostic proteomic signature for tuberculosis progression: a prospective cohort study. PLoS Med. 2019;16: e1002781. https://doi.org/10.1371/journal.pmed.1002781.

8. Donovan A, Lima CA, Pinkus JL, Pinkus GS, Zon LI, Robine S, Andrews NC. The iron exporter ferroportin/Slc40a1 is essential for iron homeostasis. Cell Metab. 2005;1:191-200. https://doi.org/ 10.1016/j.cmet.2005.01.003.

9. Tashiro K, Yamamoto M, Ushio R, Kobayashi N, Sato T, Kudo M, Kaneko T. Hepcidin exerts a negative immunological effect in pulmonary tuberculosis without HIV co-infection, prolonging the time to culture-negative. Int J Infect Dis. 2019;86:47-54. https:// doi.org/10.1016/j.ijid.2019.06.023.

10. Liang L, Liu H, Yue J, Liu L-R, Han M, Luo L-L, Zhao Y-L, Xiao $\mathrm{H}$. Association of single-nucleotide polymorphism in the hepcidin promoter gene with susceptibility to extrapulmonary tuberculosis. Genet Test Mol Biomark. 2017;21:351-6. https://doi.org/10.1089/ gtmb.2016.0300.

11. Hella J, Cercamondi CI, Mhimbira F, Sasamalo M, Stoffel N, Zwahlen M, Bodmer T, Gagneux S, Reither K, Zimmermann MB, Risch L, Fenner L. Anemia in tuberculosis cases and household controls from Tanzania: Contribution of disease, coinfections, and the role of hepcidin. PLoS ONE. 2018;13: e0195985. https://doi. org/10.1371/journal.pone.0195985.

12. Kerkhoff AD, Meintjes G, Burton R, Vogt M, Wood R, Lawn SD. Relationship between blood concentrations of hepcidin and anemia severity, mycobacterial burden, and mortality among patients with hiv-associated tuberculosis. J Infect Dis. 2016;213:61-70. https://doi.org/10.1093/infdis/jiv364.

13. Tsung SH. Several conditions causing elevation of serum CK-MB and CK-BB. Am J Clin Pathol. 1981;75:711-5. https://doi.org/10. 1093/ajcp/75.5.711.

14. Syed FF, Ntsekhe M, Gumedze F, Badri M, Mayosi BM. Myopericarditis in tuberculous pericardial effusion: prevalence, predictors and outcome. Heart Br Card Soc. 2014;100:135-9. https://doi.org/ 10.1136/heartjnl-2013-304786.

15. Tischfield JA. A reassessment of the low molecular weight phospholipase A2 gene family in mammals*. J Biol Chem. 1997;272:17247-50. https://doi.org/10.1074/jbc.272.28.17247.

16. Exeter HJ, Folkersen L, Palmen J, Franco-Cereceda A, Cooper JA, Kalea AZ, VanHooft F, Eriksson P, Humphries SE, Talmud PJ. Functional analysis of two PLA2G2A variants associated with secretory phospholipase A2-IIA levels. PLoS ONE. 2012. https:// doi.org/10.1371/journal.pone.0041139.

17. Leonarduzzi G, Gamba P, Gargiulo S, Biasi F, Poli G. Inflammation-related gene expression by lipid oxidation-derived products in the progression of atherosclerosis. Free Radic Biol Med. 2012;52:19-34. https://doi.org/10.1016/j.freeradbiomed.2011.09. 031. 
18. Chen Z, Zhao T-J, Li J, Gao Y-S, Meng F-G, Yan Y-B, Zhou H-M. Slow skeletal muscle myosin-binding protein-C (MyBPC1) mediates recruitment of muscle-type creatine kinase (CK) to myosin. Biochem J. 2011;436:437-45. https://doi.org/10.1042/BJ201 02007.

19. Xu Y-H, Deng J-L, Wang L-P, Zhang H-B, Tang L, Huang Y, Tang J, Wang S-M, Wang G. Identification of candidate genes associated with breast cancer prognosis. DNA Cell Biol. 2020;39:1205-27. https://doi.org/10.1089/dna.2020.5482.

20. Essone PN, Chegou NN, Loxton AG, Stanley K, Kriel M, van der Spuy G, Franken KL, Ottenhoff TH, Walzl G. Host cytokine responses induced after overnight stimulation with novel M tuberculosis infection phase-dependent antigens show promise as diagnostic candidates for TB disease. PLoS ONE. 2014. https://doi. org/10.1371/journal.pone.0102584.

21. Eribo OA, Leqheka MS, Malherbe ST, McAnda S, Stanley K, van der Spuy GD, Walzl G, Chegou NN. Host urine immunological biomarkers as potential candidates for the diagnosis of tuberculosis. Int J Infect Dis IJID Off Publ Int Soc Infect Dis. 2020;99:473-81. https://doi.org/10.1016/j.ijid.2020.08.019.

22. Jacobs R, Maasdorp E, Malherbe S, Loxton AG, Stanley K, van der Spuy G, Walzl G, Chegou NN. Diagnostic potential of novel salivary host biomarkers as candidates for the immunological diagnosis of tuberculosis disease and monitoring of tuberculosis treatment response. PLoS ONE. 2016;11: e0160546. https://doi. org/10.1371/journal.pone.0160546.

23. Adegbite BR, Edoa JR, Agbo PA, Dejon-Agobé JC, Essone PN, Lotola-Mougeni F, Ngwese MM, Mfoumbi A, Mevyann C, Epola M, Zinsou JF, Honkpehedji YJ, Agnandji ST, et al. Epidemiological, mycobacteriological, and clinical characteristics of smoking pulmonary tuberculosis patients, in lambaréné, gabon: a crosssectional study. Am J Trop Med Hyg. 2020;103:2501-5. https:// doi.org/10.4269/ajtmh.20-0424.

24. Russell DG, Cardona P-J, Kim M-J, Allain S, Altare F. Foamy macrophages and the progression of the human TB granuloma. Nat Immunol. 2009;10:943-8. https://doi.org/10.1038/ni.1781.

25. Chegou NN, Black GF, Loxton AG, Stanley K, Essone PN, Klein MR, Parida SK, Kaufmann SH, Doherty TM, Friggen AH, Franken KL, Ottenhoff TH, Walzl G. Potential of novel Mycobacterium tuberculosis infection phase-dependent antigens in the diagnosis of TB disease in a high burden setting. BMC Infect Dis. 2012;12:10. https://doi.org/10.1186/1471-2334-12-10.

26. Awoniyi DO, Teuchert A, Sutherland JS, Mayanja-Kizza H, Howe R, Mihret A, Loxton AG, Sheehama J, Kassa D, Crampin AC, Dockrell HM, Kidd M, Rosenkrands I, et al. Evaluation of cytokine responses against novel Mtb antigens as diagnostic markers for TB disease. J Infect. 2016;73:219-30. https://doi.org/10. 1016/j.jinf.2016.04.036.

27. Fisher ML, Kelemen MH, Collins D, Morris F, Moran GW, Carliner NH, Plotnick GD. Routine serum enzyme tests in the diagnosis of acute myocardial infarction: cost-effectiveness. Arch Intern Med. 1983;143:1541-3. https://doi.org/10.1001/archinte. 1983.00350080047012.

28. Brownlow K, Elevitch FR. serum creatine phosphokinase isoenzyme (CPK2) in myositis: a report of six cases. JAMA.
1974;230:1141-4. https://doi.org/10.1001/jama.1974.0324008002 3020.

29. Goldman J, Matz R, Mortimer R, Freeman R. High elevations of creatine phosphokinase in hypothyroidism: an isoenzyme analysis. JAMA. 1977;238:325-6. https://doi.org/10.1001/jama.1977. 03280040045018

30. Zheng J, Zheng H, Gupta RK, Li H, Shi H, Pan L, Gong S, Liang $\mathrm{H}$. Interrelationship of rotavirus infection and creatine kinase-MB isoenzyme levels in children hospitalized with acute gastroenteritis in Guangzhou, China 2012-2015. Sci Rep. 2017. https://doi. org/10.1038/s41598-017-07636-4.

31. Lakshman A, Balasubramanian P, Nampoothiri RV, Vijayvergiya R, Bhalla A, Varma SC. Elevated cardiac biomarkers and echocardiographic left ventricular dysfunction at admission in patients with dengue fever: report from a tertiary care center in Northwest India. Trop Doct. 2018;48:261-5. https://doi.org/10.1177/00494 75518785315.

32. Agudelo-Salas IY, Quinceno N, Duque J, Bosch I, Restrepo BN. Serum activity of CK and CK-MB in patients with dengue virus infection. Rev Salud Publica Bogota Colomb. 2017;19:460-7. https://doi.org/10.15446/rsap.v19n4.39597.

33. Usui A, Fujita K, Imaizumi M, Abe T, Inoue K, Matumoto S, Kato K. Determination of creatine kinase isozymes in sera and tissues of patients with various lung carcinomas. Clin Chim Acta. 1987;164:47-53. https://doi.org/10.1016/0009-8981(87)90106-9.

34. Shafi AMA, Shaikh SA, Shirke MM, Iddawela S, Harky A. Cardiac manifestations in COVID-19 patients-a systematic review. J Card Surg. 2020;35:1988-2008. https://doi.org/10.1111/jocs. 14808.

35. Han H, Xie L, Liu R, Yang J, Liu F, Wu K, Chen L, Hou W, Feng Y, Zhu C. Analysis of heart injury laboratory parameters in 273 COVID-19 patients in one hospital in Wuhan China. J Med Virol. 2020. https://doi.org/10.1002/jmv.25809.

36. Shi L, Wang Y, Wang Y, Duan G, Yang H. Meta-analysis of relation of creatine kinase-MB to risk of mortality in coronavirus disease 2019 patients. Am J Cardiol. 2020;130:163-5. https://doi. org/10.1016/j.amjcard.2020.06.004.

37. Henry BM, Benoit SW, de Oliveira MHS, Hsieh WC, Benoit J, Ballout RA, Plebani M, Lippi G. Laboratory abnormalities in children with mild and severe coronavirus disease 2019 (COVID-19): a pooled analysis and review. Clin Biochem. 2020;81:1. https:// doi.org/10.1016/j.clinbiochem.2020.05.012.

38. Harrington-Kandt R, Stylianou E, Eddowes LA, Lim PJ, Stockdale L, Pinpathomrat N, Bull N, Pasricha J, Ulaszewska M, Beglov Y, Vaulont S, Drakesmith H, McShane H. Hepcidin deficiency and iron deficiency do not alter tuberculosis susceptibility in a murine Mtb infection model. PLoS ONE. 2018. https://doi.org/10.1371/ journal.pone.0191038.

39. Nemeth E, Tuttle MS, Powelson J, Vaughn MB, Donovan A, Ward DM, Ganz T, Kaplan J. Hepcidin regulates cellular iron efflux by binding to ferroportin and inducing its internalization. Science. 2004;306:2090-3. https://doi.org/10.1126/science.1104742. 


\section{Authors and Affiliations}

Paulin N. Essone ${ }^{1,2,3}\left[\right.$ B $\cdot$ Bayode R. Adegbite ${ }^{1,2,4} \cdot$ Marien J. M. Mbadinga ${ }^{1} \cdot$ Armel V. Mbouna $^{1} \cdot$

Fabrice Lotola-Mougeni ${ }^{1}$. Ayodele Alabi ${ }^{1,5}$. Jean R. Edoa ${ }^{1,4}$ - Bertrand Lell ${ }^{1,6}$. Abraham S. Alabi ${ }^{1,2}$. Ayola A. Adegnika ${ }^{1,2,5}$. Michael Ramharter ${ }^{1,7}$. Joel F. D. Siawaya ${ }^{3} \cdot$ Martin P. Grobusch $^{1,2,4}$. Peter G. Kremsner ${ }^{1,2}$. Selidji T. Agnandji ${ }^{1,2,8}$

1 Centre de Recherches Médicales de Lambaréné, Lambaréné, Gabon

2 Institut für Tropenmedizin, Universität Tübingen and German Center for Infection Research Tübingen, Tübingen, Germany

3 Unité de Recherche et de Diagnostics Spécialisés, Laboratoire National de Santé Publique/Centre Hospitalier Universitaire Mère Enfant Fondation Jeanne EBORI, Libreville, Gabon

4 Center of Tropical Medicine and Travel Medicine, Department of Infectious Diseases, Amsterdam University Medical Centers, location Amsterdam, Amsterdam Infection \& Immunity, Amsterdam Public Health, University of Amsterdam, Amsterdam, The Netherlands
5 Department of Parasitology, Leiden University Medical Center, Leiden, The Netherlands

6 Division of Infectious Diseases and Tropical Medicine, Department of Medicine 1, Medical University of Vienna, Vienna, Austria

7 Department of Tropical Medicine, Bernhard Nocht Institute for Tropical Medicine and Department of Internal Medicine I, University Medical Center Hamburg-Eppendorf, Hamburg, Germany

8 The African Society of Clinical Trials and the African Congress for Clinical Trials, Lambaréné, Gabon 